\title{
TV/Series
}

1 | 2012

Les Séries télévisées américaines contemporaines :

entre la fiction, les faits, et le réel

\section{De Babylon à Galactica : la nouvelle science-fiction télévisuelle et l'effet-réalité}

Mehdi Achouche

\section{(2) OpenEdition \\ Journals}

Édition électronique

URL : http://journals.openedition.org/tvseries/1515

DOI : 10.4000/tvseries. 1515

ISSN : 2266-0909

Éditeur

GRIC - Groupe de recherche Identités et Cultures

Référence électronique

Mehdi Achouche, "De Babylon à Galactica : la nouvelle science-fiction télévisuelle et l'effet-réalité », TV/ Series [En ligne], 1 | 2012, mis en ligne le 15 mai 2012, consulté le 20 avril 2019. URL : http:// journals.openedition.org/tvseries/1515; DOI : 10.4000/tvseries.1515

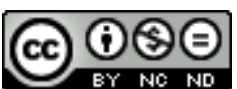

TV/Series est mis à disposition selon les termes de la licence Creative Commons Attribution - Pas d'Utilisation Commerciale - Pas de Modification 4.0 International. 


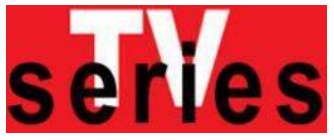

\author{
De Babylon à Galactica : \\ la nouvelle science-fiction télévisuelle et l'effet-réalité \\ Mehdi ACHOUCHE
}

\begin{abstract}
Si la science-fiction et les séries télévisées sont toutes les deux souvent pensées en termes d'évasion et de rêverie, les séries de science-fiction, plus particulièrement celles relevant du genre escapist par excellence qu'est le space opera, entretiennent depuis les années 1990 et la « renaissance » des séries télévisées une relation plus complexe et riche avec le " réel ». Les séries Babylon 5 et Battlestar Galactica surtout sont des cas d'école d'une sciencefiction thématiquement, esthétiquement et narrativement plus sophistiquée et ambitieuse, mais aussi plus sombre, que ses prédécesseurs, au sujet de laquelle la notion même de " réalisme », si elle reste ambiguë, ne paraît plus aussi incongrue que par le passé. Tirant profit des spécificités offertes par le média télévisuel, la nouvelle science-fiction entretient des rapports pluriels et dynamiques avec le(s) réel(s).
\end{abstract}

$\mathrm{U}$

n paradoxe semble sous-tendre Battlestar Galactica. D’un côté, la science-fiction (SF) est traditionnellement décrite comme le genre escapist par excellence, privilégié par Hollywood depuis une trentaine d'années. Ce genre des blockbusters regorgeant d'effets spéciaux, d'explosions et d'images grandioses nous permettrait d'avoir littéralement la tête dans la lune et dans les étoiles dans un ailleurs spatial et temporel entièrement dépaysant et divertissant. Si Hollywood et les séries télévisées sont avant tout des ouvres de divertissement censées faire oublier leur quotidien aux (télé)spectateurs, alors la science-fiction serait une d'autant plus une invitation à l'évasion loin des tracas du quotidien. La science-fiction et la télévision poursuivraient ainsi en quelque sorte le même combat.

Pourtant Battlestar Galactica est une série qui traite explicitement de sujets contemporains aussi délicats et controversés que le terrorisme, les attentats-suicides, les modalités de la résistance à une occupation étrangère, le bien-fondé de la torture de prisonniers et des lois d'exception, le fondamentalisme religieux, mais aussi plus occasionnellement l'avortement, les relations de classes, etc. Autant de thèmes qui tendent à rapprocher la série et les téléspectateurs du réel et de l'actualité (principalement le 11-septembre et les guerres en Afghanistan et en Irak, la série étant produite et diffusée de 2004 à 2009) plutôt qu'à les en éloigner et leur faire oublier leur quotidien. La mise en scène de la série tend par ailleurs elle-même à simuler le reportage de guerre ou le documentaire, et à installer cette impression générale et omniprésente de "réalisme », c'est-à-dire d'impression de réel et de réalité, plutôt que d'évasion. Le créateur de la série, Ronald 
D. Moore, va ainsi jusqu'à décrire sa création, dans un "mission statement » datant de 2003 et définissant ses objectifs créatifs pour la série, comme de la «science-fiction naturaliste ${ }^{1}$ », ce qui devrait pourtant être un oxymore et une impossibilité logique. Moore décrit sa création comme "la réinvention de la science-fiction à la télévision ». Il ajoute qu' " une nouvelle approche est requise. Cette approche consiste à introduire le réalisme dans un genre jusqu'à présent solidement irréaliste ${ }^{2} »$.

Néanmoins ce phénomène n'est pas entièrement nouveau, et s'il n'a jamais été aussi prégnant que dans Galactica, la tendance à la SF de télévision naturaliste ou " réaliste » est bien une vague de fond dont on peut trouver les premiers signes dans les années quatre vingt-dix. C'est principalement le cas dans la série Babylon 5, qui est en quelque sorte à la science-fiction télévisuelle ce que Homicide: Life on the Streets (NBC, 1993-1999) et NYPD Blue (ABC, 1994-2005) sont à la série policière, ou bien $E R$ (Urgences) (NBC, 1994-2009) à la série médicale - c'est-à-dire une renaissance pour le genre, une régénération thématique et esthétique qui est celle des séries télévisées américaines dans leur ensemble au début de la décennie. Diverses techniques empruntées au cinéma (travelings et plans-séquences, caméras portées, angles de prise de vue atypiques et alternés, montage serré, jeu sur les ombres et lumières, etc.), mais aussi une nouvelle exigence scénaristique, un plus fort développement des personnages et la réapparition d'une narration feuilletonnante, contribuent à accroître la popularité et à légitimer les séries télévisées. Cette légitimation se fonde sur un rapport perçu comme plus étroit avec le « réel ».

L'effet de ces changements est en effet fréquemment d'accroître l'impression de réalité qui se dégage de séries qui, policières, médicales ou judiciaires, font mine de parler du quotidien d'individus qui appartiennent eux-mêmes au quotidien des téléspectateurs. Mais qu'en est-il lorsque les mêmes techniques sont appliquées à la science-fiction, supposée battre en brèche ce même quotidien, et qui repose sur une notion, l'imaginaire, qui se définit justement contre le « réel »? Plus que de « réalisme », il est possible de parler d' « effet-réalité » ou, pour reprendre l'expression de Roland Barthes, d' « effet de réel 3 » : un effet - thématique, esthétique, narratif - qui dépasse le seul vraisemblable propre à un genre pour une référentialité cette fois omniprésente et structurante. Dans le même temps le terme d'effet-réalité est suffisamment vague pour laisser ouverte la question de l'efficacité et de

1 Tiffany Potter, C.W. Marshall, Cylons in America - Critical Studies in Battlestar Galactica, New York, Continuum, p. 5. Ronald D. Moore, «Battlestar Galactica: Naturalistic Science Fiction or Taking the Opera out of Space Opera ", $<$ http://en.battlestarwiki.org/wiki/Naturalistic_science_fiction>, dernier accès le 30/10/2011.

2 Ibid.

3 Roland Barthes, « L'effet de réel », in Communications, 11, 1968, 84-89, passim. 
la finalité d'un tel effet - la nature exacte du rapport du genre au réel d'un côté, et la nature précise du réel en question de l'autre. Sans être nécessairement « réalistes », ces séries donnent l'impression d'un rapport beaucoup plus étroit au réel qu'auparavant, caractéristique fondamentale de la «renaissance » des séries télévisées. Si des différences importantes existent entre Babylon 5 et Battlestar Galactica, les deux séries sont néanmoins capitales dans l'évolution et la maturation du genre, qui gagne en sophistication avec le temps et dont le rapport au réel et au présent se fait progressivement plus complexe, riche et ambivalent. Les thématiques abordées par ces séries, leur mise en scène et enfin le mode narratif qu'elles adoptent rompent ainsi clairement avec le passé et contribuent tous à cet effetréalité d'un genre nouveau.

\section{La dystopie des étoiles, ou la satire du space opera}

La science-fiction est un genre particulièrement riche et exubérant, mais Babylon 5 et Galactica relèvent d'un sous-genre ou d'une variété de science-fiction central à la télévision américaine depuis soixante ans, le space opera. Or, ce sous-genre est précisément celui qui est le plus souvent décrit comme la variété la moins solide et sérieuse de la SF, son avatar le plus juvénile et partant le plus irréaliste - et paradoxalement, son versant le plus idéologisé. C'est aussi précisément contre ce sous-genre que Moore s'insurge dans son essai, contre « ses personnages clichés, son techno-langage de pacotille, ses extraterrestres aux grosses têtes, son cabotinage, son héroïsme creux $^{4} \gg$. Le space opera constituerait la cristallisation de tous les défauts traditionnellement associés à la SF, voire aux séries télévisées le terme "space opera » est après tout dérivé de celui de " soap opera », un des genres les plus stigmatisés du petit écran 5 . Or le genre va peu à peu évoluer vers moins d'escapism et d'idéologie pour plus d'emprise avec le réel et un regard plus ambivalent sur celui-ci.

Les premières itérations du genre à la télévision prêtent effectivement le flanc à la critique. Dès les années cinquante, les vaisseaux spatiaux et leurs ponts de commandement, la lutte de cryptomilitaires américains dans l'espace contre des extraterrestres totalitaires, et l'exploration de planètes exotiques, les tropes majeurs du space opera, se multiplient à la télévision, dans des séries systématiquement destinées à un jeune public : Captain Video and His Video Rangers, créé dès 1949 (1949-1955), Space Patrol (1950-1955), Tom Corbett, Space Cadet (1950-1955), Buck Rogers (1950), Rod Brown of the Rocket Rangers (1953-1954), Flash Gordon (1954-1955),

${ }^{4}$ Moore, op. cit

5 John Clute, Peter Nicholls, The Multimedia Encyclopedia of Science Fiction, CD-Rom (édition pdf), Grolier Multimedia, 1995, p. 2193-2195. 
dont les seuls titres soulignent la parenté au Western. Ces séries proviennent souvent directement des serials et des séries radiophoniques des années trente, jusqu'au dernier et plus célèbre d'entre eux, Star Trek, dans les années soixante (1966-1969)6. L'idée de voir des militaires américains explorer des planètes étrangères et affronter des civilisations extraterrestres opposées aux valeurs humanistes de l'Amérique7, schéma que l'on retrouve fréquemment dans le cinéma de la décennie, reste d'ailleurs populaire aujourd'hui, comme le montre le succès contemporain des franchises Star Trek et surtout Stargate, même si comme on le verra l'influence du genre militaire est plus prégnante aujourd'hui que celle du Western.

La forme est d'autant plus séduisante pour la télévision qu'elle se prête parfaitement à une déclinaison hebdomadaire, chaque semaine introduisant une nouvelle planète, une nouvelle civilisation ou un nouveau phénomène naturel à explorer, affronter et surmonter 8 . Ici le réel n'a que très peu droit de cité, l'emphase étant sur l'exotisme des mondes et des civilisations visitées, ainsi que la façon dont celles-ci sont opposées à l'humanité et surtout aux Etats-Unis - chaque nouveau monde doit apporter la confirmation que le modèle américain n'a effectivement pas d'égal. La grandeur de ce modèle est le plus souvent symbolisée par l'équipage au centre de la série, dont Star Trek est l'exemple emblématique: parfaitement hiérarchisée, homogène et soudée, l'équipe est composée d'hommes et de femmes qui se battent pour imposer les valeurs humanistes et progressistes de la nation, tout en faisant perdurer l'idéal d'une Frontière et d'un progrès éternels.

Quelques tentatives sont pourtant tentées, avant les années quatre vingt-dix, pour transformer le genre, pour l'amener notamment vers les territoires plus sombres et moins triomphalistes de la dystopie - c'est-à-dire un futur (parfois un passé) et une société cauchemardesques où des héros doivent typiquement combattre pour leur survie et cherchent à fuir leurs persécuteurs, plutôt que jouer les redresseurs de torts hebdomadaires. Le cinéma de science-fiction se fait ainsi dès le début des années soixante-dix beaucoup plus sombre et

\footnotetext{
${ }^{6}$ M. Keith Booker, Science Fiction Television, Westport, Praeger Publishers, p. 5. Mais il est vrai que dans le même temps étaient créées des séries s'éloignant du space opera et proposant des scenarios plus sophistiqués et originaux, principalement les séries anthologiques, notamment Tales of Tomorrow (1951-1953), Science Fiction Theater (19551957), The Twilight Zone (1959-1964), et The Outer Limits (1963-1965), qui toutes à l'occasion produisirent des épisodes relevant du space opera (Booker, p. 5-6).

7 La découverte sur d'autres planètes de régimes crypto-communistes est d'ailleurs un grand classique de la science-fiction depuis le tournant du XXe siècle, avant que les néonazis ne fassent leur apparition sur d'autres planètes dès les années 1940. En utilisant les Cylons comme alter-ego imaginaires des fondamentalistes et terroristes religieux, Galactica se situe donc dans un schéma SF classique, même si son traitement de l'opposition se révèlera beaucoup moins manichéen que ses prédécesseurs.

${ }^{8}$ On peut ainsi partiellement expliquer que le space opera soit très peu présent sur le grand écran dans les années cinquante.
} 
pessimiste, absorbant très vite l'impact de la contre-culture, de l'opposition à l'establishment et de la remise en cause du progrès scientifique et technologique. Le cinéma de science-fiction se révèle particulièrement apte à représenter et problématiser les thématiques et inquiétudes de l'époque : il met en scène des sociétés futuristes rongées par la surpopulation (Soylent Green, 1973), la pollution et la déforestation (Silent Running, 1972), la guerre nucléaire et ses retombées (Planet of the Apes, 1968; A Boy and his Dog, 1975), ou devant affronter leurs propres créations technologiques (THX 1138, 1971 ; Logan's Run, 1976). Mais la télévision est beaucoup plus rétive à ce genre de schéma, et toutes les tentatives d'adaptation des succès contemporains du grand écran par CBS (comme les adaptations TV Planet of the Apes (1974), Logan's Run (1977) et Beyond Westworld (1980), ou encore le remake dystopique de Buck Rogers, 1979-1981) se soldent par des échecs d'audience. La première mouture de Galactica (ABC, 1978-1979) elle-même, dont tout le monde s'attend à ce qu'elle soit un grand succès étant donné le triomphe récent de Star Wars (dont la série est très clairement inspirée, notamment dans son esthétique, comme nous le verrons), se solde par un échec cuisant 9 .

Il faut donc attendre le début des années quatre vingt-dix et la «renaissance» des séries télévisées pour voir le space opera de télévision connaître enfin sa propre renaissance, cette fois sur un mode dystopique, emboîtant finalement le pas au cinéma et à la littérature. Le mode dystopique n'est pas nécessairement plus réaliste que le schéma précédent, il conduit néanmoins les nouvelles séries à abandonner l'idéologie propre au genre, à traiter de thématiques politiques et sociales inconnues à leurs prédécesseurs, et à resserrer les liens qui les unissent au monde "réel » et particulièrement à son actualité. La science-fiction, née en au XIXe siècle en même temps que la fiction historique et l'historiographie moderne, est un genre tout entier axé sur l'Histoire, où le futur sert souvent à représenter et à problématiser l'Histoire passée et présente - l'Histoire en train de se nouer, la SF permettant d' "appréhender le présent en tant qu'histoire ${ }^{10}$ ", comme l'écrit Fredric Jameson. Le space opera traditionnel représente la version mythifiée de l'Histoire - le progrès et

\footnotetext{
9 Il faut néanmoins préciser ici que tous les grands succès de space opera de télévision actuels, des Stars Trek aux Stargate en passant par Babylon 5 et Galactica, sont diffusés sur la syndication et/ou le câble, et peuvent donc se contenter d'une audience moindre. La nouvelle itération de Galactica est ainsi diffusée sur SyFy, chaîne du câble, tandis que l'original était diffusé sur ABC, un des grands networks du pays. On peut également ici mentionner la série $V$ (1984-1985), qui par le degré avec lequel elle fait écho à la Deuxième guerre mondiale, l'occupation d'un pays ennemi et les problèmes posés par la résistance et la collaboration avec l'ennemi, ainsi que la figure de l'hybride et d'une possible réconciliation ultime entre les deux espèces, annonce les séries ultérieures (cf. infra). Mais $V$ comme ses prédécesseurs ne dépasse pas le stade de la première saison.

${ }^{10}$ Frederic Jameson, Archeologies of the Future, The Desire Called Utopia and Other Science Fictions, Londres, Verso, 2007, p. 288.
} 
la croissance éternels - tandis que sa nouvelle déclinaison tente de rendre compte d'une Histoire plus immédiate et agitée, qui rend particulièrement compte des tourments du XXe siècle en représentant ceux-ci de façon détournée.

C'est le cas en 1994 avec le lancement de Babylon 5 (B5), dont les points communs avec Galactica sont particulièrement nombreux et probablement pas tous accidentels. $B_{5}$ innove tout d'abord en étant l'une des premières séries à situer son action à bord d'une station spatiale. Elle abandonne l'exploration et le schéma dynamique d'une Frontière éternelle qui faisait l'âme du space opera pour adopter un schéma statique où ce n'est plus l'exploration et la découverte de l'inconnu qui importent, ni la critique de civilisations barbares ou décadentes, mais la cohabitation interraciale, la diplomatie et la realpolitik. Une fois l'excitante conquête achevée, il faut bien gérer les nouveaux territoires et les peuples qu'ils abritent. Le mythe romantique de la conquête cède donc la place à une représentation plus nuancée et sobre, voire pessimiste, où l'Empire / Fédération multiethnique et multiculturel ne s'accroît plus tant qu'il cherche à assurer sa difficile cohésion interne ${ }^{11}$.

B5 choisit de dramatiser les innombrables difficultés politiques d'un futur intergalactique construit sur l'exagération, le grossissement des tendances et phénomènes de la décennie. La station Babylon est représentée comme une sorte de Nations Unies, ou alternativement une cour des miracles des étoiles, reflétant le chaos et les violences interethniques qui ensanglantent les années quatre vingt-dix et l'immédiat après-guerre froide. La série choisit dans le même temps d'y superposer sa propre guerre froide de l'avenir, faisant l'apologie de la Ligue des Mondes Non-Alignés qui tentent d'imposer une troisième voie aux deux superpuissances de la galaxie, toutes deux

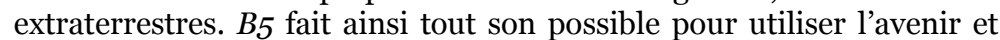
l'anticipation dystopique pour refléter le passé ainsi que les dangers et les défis posés par le présent, et prend ses distances avec l'idéal utopique américaniste pour une vision plus authentiquement multiethnique et multiculturelle, pour le meilleur ou pour le pire.

La série peut également aborder un des thèmes chers à la science-fiction visuelle depuis au moins Star Wars, le risque de voir un gouvernement démocratique être renversé ou dévoyé par un régime totalitaire. C'est le cas durant les troisièmes et quatrièmes saisons, où

${ }^{11}$ Il est vrai que le pilote de la série est diffusé quelques semaines après celui de Star Trek: Deep Space Nine (Syndication, 1993-1999), qui lui-aussi se situe à bord d'une station spatiale et traitera de thématiques très similaires à celle de $B_{5}$. Mais ce nouveau Star Trek est au départ très similaire aux précédentes séries de la franchise, et c'est seulement en 1995 , avec le lancement de sa quatrième saison, que la série commence vraiment à se rapprocher de $B 5$, sans doute sous l'influence de cette dernière. L'infléchissement sombre et partiellement dystopique de Star Trek est par ailleurs le marqueur le plus spectaculaire de l'évolution de la SF télévisuelle dans les années quatre vingt-dix. 
l'équipage de la station doit faire face à l'assassinat du président, l'avènement d'un régime autocratique sur Terre à l'instigation du viceprésident, et la suppression graduelle des libertés individuelles, avant de devoir finalement déclarer son indépendance et lutter pour la démocratie. Les intrigues multiplient la même noirceur politique au cours des cinq saisons de la série, revenant toujours et encore à cette question centrale qu'est la bonne gouvernance, la défense de sa culture et de sa civilisation face aux multiples dangers extérieurs et, surtout, la défense de la démocratie face aux menaces endogènes, c'est-à-dire les mêmes thématiques que Galactica ${ }^{12}$.

Cette dernière se situe certes à bord de vaisseaux spatiaux en constant mouvement du fait de la traque organisée par les Cylons. La plupart des épisodes ne jouent cependant pas sur cette dynamique et utilisent les vaisseaux de la flotte, surtout le croiseur Galactica, comme ils pourraient utiliser une station spatiale statique (les deux séries, Babylon 5 et Battlestar Galactica, tirent d'ailleurs leur titre du nom de la structure spatiale qui est au centre de leurs intrigues). L'exploration ne joue qu'un rôle infime dans les deux séries, et la figure de l'extraterrestre disparaît même entièrement de Galactica, qui renonce à toute forme d'exotisme $\mathrm{SF}$ au profit d'un huis-clos entre les personnages. Ceux-ci n'auront de cesse au cours des quatre saisons de s'affronter les uns aux autres au gré d'alliances évanescentes, illustrant les divisions qui minent une société qui s'avère être en réalité son premier ennemi - la belle cohésion sociale du passé n'est plus de mise.

A l'inverse la politique y prend une place considérable, la série étant cette fois considérablement influencée par le 11-septembre et les nombreuses questions éthiques posées par l'événement. L'argument SF apparaît alors comme une stratégie narrative de contournement permettant d'aborder le sujet de manière qui ne soit pas trop frontale et traumatisante. La série se concentre notamment sur la réponse apportée par les autorités civiles et militaires aux attaques extérieures et le risque de voir ces autorités, prêtes à sacrifier les libertés individuelles sur l'autel de la sécurité nationale, trahissant ainsi la nature démocratique de la société du futur. La présidente Roslin et les choix parfois moralement discutables, voire franchement amoraux, qu'elle prend illustrent cette thématique, de même que l'élection du vice-président Baltar, qui s'impose à la tête des colonies à grands coups de populisme à la fin de la saison deux.

De plus, la série explore les deux religions concurrentes de ce monde alternatif, qui font volontairement écho, la première aux religions polythéistes païennes (certains personnages s'appellent ou

${ }_{12}$ Il n'est donc pas surprenant de constater la présence dans l'équipe de $B 5$ comme consultant du célèbre auteur SF Harlan Ellison, un des principaux auteurs dystopiques des années 1960 / 1970 et lui-même créateur d'une série relevant de la "nouvelle sciencefiction » des années 1970, The Starlost (Syndication, 1973-1974). 
sont surnommés Appolo, Athena, etc., les douze planètes coloniales sont nommées d'après les douze constellations grecques, etc.), la seconde au christianisme, tout autant que Babylon 5 pouvait s'attarder sur les croyances et rites religieux de ses nombreuses cultures extraterrestres ou terrestres (cf. par exemple le « Fondationnisme ${ }^{13}$ » terrestre). Là encore Galactica appartient à son époque, se concentrant sur le fanatisme religieux, allant jusqu'à mettre en scène un attentatsuicide commis à bord du métro de Caprica dans l'épisode pilote de la série éponyme (Syfy, 2009-2010), spin-off de Galactica. La référentialité de Galactica va ainsi beaucoup plus loin que celle de $B_{5}$, la science-fiction n'hésitant plus ici à surligner les parallèles qui l'unissent au monde « réel ».

Les deux séries utilisent donc leurs sociétés spéculaires du futur pour mettre en scène des questions politiques, éthiques et sociales pérennes et dans le même temps contemporaines. Elles font ainsi paradoxalement œuvres de science-fiction parmi les artefacts culturels les plus révélateurs de leurs époques respectives. Malgré la présence d'ennemis extérieurs, les deux séries mettent un point d'honneur à souligner à quel point le véritable danger est endogène à la démocratie, et en cela le chemin parcouru par Galactica durant ses quatre saisons, de l'ennemi robotique extérieur à la découverte qu'une partie de l'équipage et de la distribution sont eux-mêmes des Cylons à la fin de la saison trois, jusqu'à la décision finale d'arrêter la guerre et de cohabiter en paix, est exemplaire. D'autant plus que les machines, au contraire des aliens, présentent l'avantage scénaristique d'être la création de l'humanité, ce qui permet à la série d'insister d'autant plus sur les responsabilités de celle-ci dans ses propres mésaventures, ainsi que sur son hubris scientifique et technologique.

Ni située dans le futur ni vraiment dans le passé, malgré les révélations finales de la série, on peut considérer que Galactica se situe en fait dans une sorte d'univers parallèle au nôtre, un décalage finalement très léger dont la représentation formellement et thématiquement «réaliste » est censée être le marqueur explicite, soulignant au spectateur à quel point le monde représenté est proche du sien. L'uniforme des militaires de l'avenir a beau être distinct, il ressemble à un uniforme militaire actuel ; la présidente de cette société parallèle voyage à bord non pas d'Air Force One mais de Colonial One ; le métro sur Caprica ressemble fortement au métro américain standard tout en ayant un aspect vaguement plus futuriste ${ }^{14}$; et la religion des humains et des Cylons rappelle très fortement les religions humaines

13 Le « Fondationnisme » est une religion ou spiritualité syncrétique née sur Terre suite aux premiers contacts extraterrestres et qui constitue un des aspects les plus utopiques de la série. Elle cherche à identifier les sources et caractéristiques communes des religions terrestres afin d'en proposer la fusion idéale et épurée de toute pollution historique et dogmatique.

${ }^{14}$ Dans l'épisode pilote de Caprica (1.0). 
historiques, tout comme l'histoire de Caprica et des douze colonies rappelle celle de la Terre. Galactica illustre à un tel point le fonctionnement de la science-fiction qu'elle en est comme le paroxysme, manœuvrant habilement sur la frontière séparant le réel de son reflet imaginaire, déformant juste assez la réalité pour en donner un aperçu modifié mais intelligible, permettant sans grande difficulté de remonter jusqu'à la source référentielle.

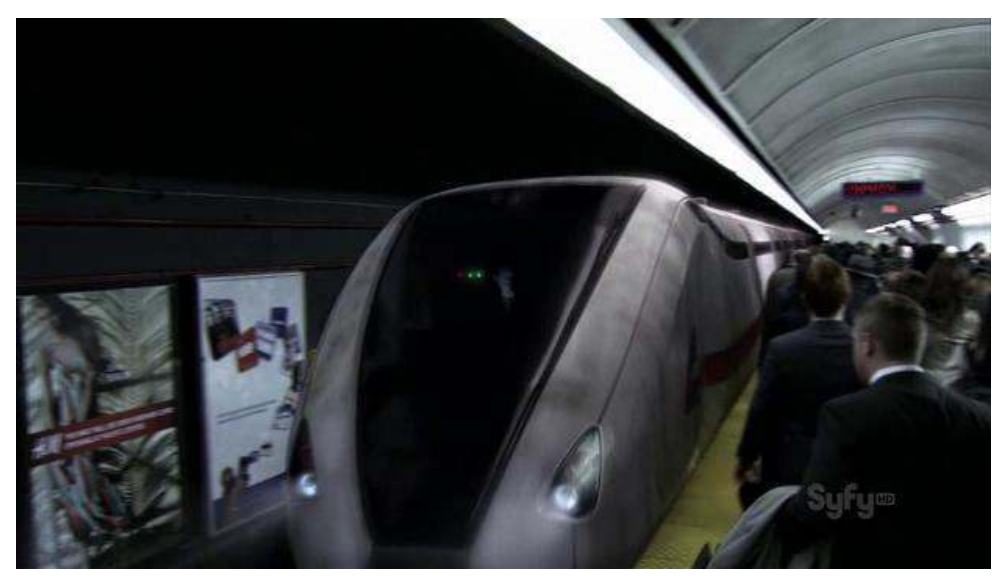

Dans le même temps la figure de l'hybride prend une importance croissante tout au long de la série, permettant d'ouvrir une perspective utopique pour le futur, l'après-fin de la série, lorsque les deux espèces / " races » pourront enfin vivre ensemble en paix. Un parcours qui prend d'autant plus de résonances dans le contexte post11 septembre et les peurs de "guerres entre civilisations" d'un Huntington. Le space opera, ses nombreuses planètes qui sont tout autant de nations distinctes, et ses civilisations se révèlent alors le sous-genre parfait pour mettre en scène et problématiser les questions posées par le moment présent, et en cela l'effet-réalité joue à plein : témoin de leur temps, ces séries font tout pour en revenir au réel, pour que la réflexion soit le moins métaphorique ou obscure possible et que le téléspectateur puisse raccorder ce qu'il voit à son environnement immédiat et sa propre expérience. En science-fiction et plus particulièrement dans Galactica (comme l'explicite le series finale), le futur se rapproche du passé au point de s'y confondre - l'avenir n'est souvent que le moyen détourné de mettre en scène le passé plus ou moins proche (les conflits mondiaux, le fascisme, la Guerre froide, le 11-septembre) et in fine le présent. C'est en cela que l'on peut comprendre la notion de réalisme appliquée à la science-fiction et au space opera - l'idée que les mondes imaginaires typiques du genre 
sont non seulement des invitations ludiques à la rêverie, mais aussi des moyens détournés précieux pour mettre à distance le réel et ainsi mieux l'envisager. C'est ce que le théoricien de la SF Darko Suvin appelle la « distanciation cognitive » (« cognitive estrangement ${ }^{15}$ »), où la distance acquise permet de jeter un regard neuf, « défamiliarisé », sur le réel et la société, mécanisme que l'on peut associer à l'effetréalité - la capacité des deux séries à s'éloigner de la réalité pour mieux la contourner et y revenir par un chemin indirect. Comme l'écrit encore Suvin, la SF (une certaine SF en tous les cas) use de «l'imagination comme un moyen pour comprendre les tendances latentes dans la réalité », $B 5$ et Galactica montrant en quoi la télévision peut désormais faire sienne ce mécanisme spécifique.

\section{Une nouvelle esthétique}

L'effet-réalité est aussi esthétique, la rupture la plus flagrante et immédiate opérée par Galactica reposant dans une mise en scène qui n'a plus grand-chose à voir avec ses prédécesseurs et renforce le sentiment de réalisme émanant de la série. Pourtant c'est peut-être ici que l'effet-réalité est le plus artificiel.

Babylon 5 tranchait déjà avec les décors lisses, les vêtements et l'éclairage neutre typiques de Star Trek, se rapprochant à nouveau plus d'une esthétique apparue au tournant des années quatre-vingt via principalement trois films : le premier Star Wars (1977), Alien (1979) et Blade Runner (1982). Tous trois imposent une sensibilité esthétique sombre et presque décadente, au diapason de leurs récits dystopiques - plans beaucoup moins exposés à la lumière qu'auparavant, utilisation de couleurs sombres ou délavées, matériel, décors urbains et costumes sales, usés et tout en aspérités, etc.. Le résultat est une " esthétique de l'entropie » où " tout a l'air vieux, comme si cela faisait très longtemps que c'est là ${ }^{16}$ ». La même poussée esthétique se retrouve dans $B_{5}$, où certaines machines ou sections de la station ne fonctionnent plus ou n'ont pas pu être achevées, où les denrées alimentaires et les matières premières sont limitées et parfois rationnées, où le matériel s'use, etc. Galactica va plus loin encore dans cette direction, faisant le choix d'un certain rétro-futurisme ${ }^{17}$, c'est-à-dire en mettant en scène des technologies qui n'ont rien de particulièrement avancées, voire sont

\footnotetext{
15 Darko Suvin, Metamorphoses of Science Fiction, Londres, Yale University, 1979, p. 4-6. ${ }_{16}$ " everything looks old, like it's been around for a while", remarque cité in Vivian Sobchack. Screening Space. The American Science Fiction Film. New Brunswick, Rutgers University Press, $2^{\mathrm{e}}$ ed., 2004, p. 263.

17 Il existe plusieurs acceptions du terme ; la principale « propose de donner 'vie' aux visions $\mathrm{du}$ futur telles que l'imaginait la culture populaire autrefois " (Etienne Barillier, Steampunk!, Lyon, Les Moutons électriques, 2010, p. 15), tandis que nous l'utilisons ici pour signifier la présence anachronique dans le futur représenté d'éléments désuets appartenant au passé du téléspectateur.
} 
parfois obsolètes dans le présent des téléspectateurs (cf. par exemple la radio dans l'avant-dernier épisode de la saison trois, ou les micros utilisés tout au long de la série par la presse et les politiques pour faire leur discours, qui rappellent fortement les années 1930-40): si les vaisseaux spatiaux et le voyage à la vitesse de la lumière existent bien, les vaisseaux en question ressemblent volontairement plus à des croiseurs contemporains ou même surtout (dans leur intérieur) à des sous-marins (pas de grandes baies vitrées, de nombreuses coursives étroites, de lourdes portes métalliques qui ne s'ouvrent pas automatiquement, des écoutilles, etc. $\left.{ }^{18}\right)$. Pas de téléporteurs ni de champ de force, pas même d'Internet, peu d'ordinateurs ou d'écrans de contrôle, des téléphones filaires massifs, uniquement des armes à feu traditionnelles, ce qui conduit au paradoxe d'une série de sciencefiction beaucoup moins "technologiste" que de nombreuses séries policières actuelles censées se passer dans le présent (Les Experts, NCIS, Bones, etc. ${ }^{19}$ ). Le réalisme visuel de la série, qui brouille la temporalité précise du récit, est donc particulièrement frappant pour un genre où le « réalisme » est normalement jugé à l'aune du degré d'extrapolation et de véracité scientifique et technologique exhibé par l'œuvre en question : situé dans un avenir dont la relation historique et géographique au nôtre est loin d'être évidente, Galactica met en scène très peu de ces technologies dont on peut fantasmer un jour l'existence dans la réalité. Le réalisme "prédictif » n’y a donc quasiment plus cours.

\footnotetext{
${ }^{18}$ Ronald D. Moore, grand amateur de la marine militaire, revendique d'ailleurs dans ses podcasts cette analogie, expliquant s'être notamment inspiré du film de guerre Crimson Tide (1995) ainsi que des films de la deuxième guerre mondiale, tandis que les téléphones filaires massifs présents à bord du vaisseau proviennent d'un ancien destroyer de la marine canadienne (John Hodgman, « Ron Moore's Deep Space Journey », New York Times, 17/05/2005).

${ }_{19}$ L'explication est en partie scénaristique, puisque Galactica est après tout une série postapocalyptique, où les trésors technologiques du passé ont été en grande partie détruits, et c'est d'ailleurs en grande partie du fait de sa relative obsolescence technologique que le Galactica a échappé à l'apocalypse du tout début de la série.
} 


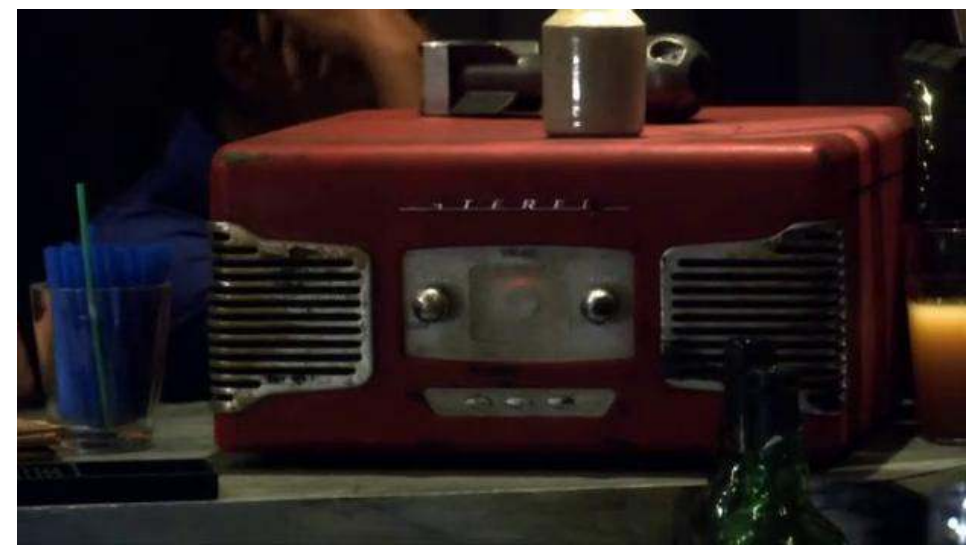

Dans le même temps, la série se protège d'un des reproches les plus souvent adressés à la science-fiction - celui de s'intéresser plus aux gadgets qu'aux histoires et aux personnages - et se rapproche du réel grâce à des technologies qui existent ou ont bel et bien existé dans le quotidien du spectateur. Le " réalisme » consiste donc ici à esquiver le futurisme et les tropes qui tendent habituellement à définir et réifier un genre, afin de «naturaliser» celui-ci et le rendre plus immédiatement proche et accessible au téléspectateur ${ }^{20}$.

Mais si le « réalisme » traditionnel de la SF n'est pas pertinent ici, celui relatif au genre militaire est plus que jamais présent. Car Galactica relève donc de la science-fiction dans son versant militaire, pour ne pas dire militariste, qui suit le quotidien de soldats « au front », leurs relations et tensions, leur difficile intimité, leur gestion du stress post-traumatique, l'organisation hiérarchique plus ou moins rigide, le paternalisme traditionnel de l'institution, la place des femmes au sein de l'organisation, etc. Même les combats spatiaux rappellent souvent des combats aériens traditionnels, et les pilotes qui sont au cœur de la série (le Galactica est à la fois un croiseur et un porte-avions de l'espace) ont souvent d'ailleurs des répliques empruntées à des films de guerre, de Patton à Top Gun. A tel point que l'on peut facilement oublier, lors de nombreuses scènes d'intérieur, que l'on est dans une série de science-fiction. Galactica est même peut-être l'unique série produite depuis le 11-septembre proposant un regard sur le quotidien des marines engagés sur le front qui a dépassé le stade de la première

\footnotetext{
20 L’influence du cinéma se fait à nouveau sentir ici, soulignant une fois encore la sophistication croissante du genre. Des films comme Blade Runner (1982) et Gattaca (1997) utilisent le même type de rétro-futurisme, cherchant déjà à échapper au piège générique d'une $\mathrm{SF}$ « gadgetophile ».
} 
saison $^{21}$. Ici la notion d'effet-réalité tend à se confondre avec l'idée de " suivre et décrire le quotidien de... », répondant à une acception toute moderne, et essentiellement télévisuelle, de la notion de « réalisme ». Comme l'écrit Moore dans son essai :

Un téléspectateur devrait pouvoir avoir l'impression pendant un moment qu'il vient de zapper accidentellement sur un documentaire de 60 Minutes [plus ou moins l'équivalent d'Envoyé Spécial] racontant la vie quotidienne à bord d'un porte-avions, jusqu'à ce que quelqu'un se mette à parler de Cylons et de vaisseaux spatiaux.

La mise en scène simulant le reportage ou le documentaire prend alors tout son sens, cherchant à simuler le reportage de guerre, les images tournées par les embedded journalists à partir de 2003 en Irak et devenues iconiques de ce conflit. Le réalisme en tant que tentative de reproduction et de mimétisme du réel joue donc ici à plein. Galactica innove clairement par rapport à Babylon 5, dont la mise en scène était de ce point de vue beaucoup plus conventionnelle, et apparaît au final comme la première série de science-fiction à véritablement faire un usage original de la caméra, phénomène qui là encore s'est répandu parmi les séries télévisées à partir du début des années quatre vingt-dix (Homicide, The X-Files, Urgences), mais pour une mise en scène plus caractéristique du "réalisme » tel qu'il est perçu depuis le début des années 2000, un réalisme de télévision (et ici 24 heures chrono, qui aborde des thématiques similaires à Galactica, est sans doute une influence aussi importante que les images rapportées d'Irak).

Le paradoxe est que ce réalisme est obtenu non pas par un effacement de la mise en scène afin de « laisser passer le réel » avec le moins de filtres possibles comme chez les néoréalistes italiens, mais au contraire par le caractère ostentatoire de la mise en scène, sa prégnance à l'écran, la fréquence avec laquelle la caméra bouge ou tremble légèrement, zoome soudainement, insère des gros plans ou choisit des angles de prise de vue inattendus, la présence sur la bandeson des transmissions radios entre des soldats hors-champs, etc. (Moore fait d'ailleurs référence au cinéma-vérité dans son essai). Le naturalisme dont parle Moore consiste donc à copier un certain de type de mise en scène journalistique typique de la télévision et du grand reportage. Le réalisme recherché tend alors à se confondre avec l'effetréalité produit par une mise en scène qui simule un autre type spécifique de mise en scène désormais associé dans l'esprit des

${ }^{21}$ On a souvent remarqué, pour expliquer l'échec de ces productions, que le public américain ne désirait pas voir dans ses fictions ce qu'il voyait déjà quotidiennement au journal télévisé. La stratégie de l'éloignement typique de la science-fiction aurait donc peutêtre permis à Galactica de contourner cette réticence. 
téléspectateurs à une réalité prise sur le vif, un «néo- néoréalisme » qui prend en fait le contre-pied de son prédécesseur. L'effet-réalité utilisé dans Galactica correspondrait donc à la réification d'une mise en scène qui aurait perdu sa spontanéité initiale, est utilisée comme un effet spécial et menace de se transformer en gimmick insignifiant, ou qui entretiendrait l'illusion d'une absence presque totale de médiation. Ici le réalisme correspond donc à un nouveau canon esthétique qui dicte les règles du nouvel effet-réalité, tout aussi conventionnel que le précédent. Il s'agit donc bien d'un réalisme de télévision, les images typiques du JT ou d'un CNN, exemple saisissant de télévision s'autophagocytant, où fiction et réalité s'entremêlent presque inextricablement, phénomène de fond mais frappant lorsqu'il s'agit du genre escapist par excellence qu'est supposé être la science-fiction. Le paradoxe est donc ici que cette esthétique représente peut-être l'aspect le plus artificiel du show, où l'effet réalité tend à se muer en effet spécial et en effet de manche.

Le paradoxe de voir employer l'effet-réalité dans le cadre d'une série de science-fiction s'explique dans la mesure où ce « réalisme de télévision » peut se marier avec un univers de science-fiction puisque tous deux relèvent de la re-création du réel, auquel participe bien la science-fiction, plutôt que de vouloir organiser notre évasion du pénitencier que serait le réel. Le phénomène le plus emblématique de ce paradoxe est l'importance de la technologie et de la postproduction dans la simulation du réel par la série, puisque des caméras et des effets spéciaux digitaux sont utilisés pour créer en postproduction la plupart des zooms, recadrages, mises au point abruptes et tressautements caractéristiques de la série ${ }^{22}$. Là encore Babylon 5 a ouvert la voie, étant la première série à faire un usage systématique des images et des trucages digitaux. Mais dans Galactica la technologie est d'autant plus importante qu'elle se fait discrète et tend non à émerveiller à l'aide des traditionnelles batailles spatiales (dont la série n'est certes pas dénuée) mais à créer un effet de réel qui n'a jamais été aussi artificiel et médiatisé. Un phénomène qui rappelle la notion de « photoréalisme » si importante aujourd'hui dans le cinéma de sciencefiction, et qui montre à quel point la réalité et le réalisme peuvent devenir un effet spécial, un trucage ajouté en post-production. Comme l'écrit Moore :

Nos vaisseaux seront mis en scène comme de vrais vaisseaux que quelqu'un a dû partir filmer avec une vraie caméra. Cela veut dire pas de plans héroïques en $3 \mathrm{D}$ qui effectuent des

$22<$ http://www.effets-speciaux.info/article?id=206>, consultation le 20/04/2011. Cf. aussi <http://www.youtube.com/watch?v=zz_QGYqmjQI>, (consultation le 25/o6/2011), pour une série de vidéos de démonstration de l'utilisation du logiciel en question, LightWave $3 \mathrm{D}$, pour les besoins de la série. 
travelings and des zooms incroyables sur un simple clic de souris. Les questions que nous nous poserons avant de réaliser chaque plan digital seront du type : "Comment est-ce qu'on a tourné ce plan? Où est placée la caméra ? Qui la tient ? Est-ce que le cameraman est dans un autre appareil ? Est-ce que la caméra est installée sur l'aile?

Le réalisme esthétique recherché par la série consiste donc à aller à l'encontre des pratiques habituelles afin à nouveau de renforcer l'effet-réalité sur lequel se fonde la série. Si les technologies mises en scène par la série doivent être le plus proche possible de la réalité des téléspectateurs (leur présent ou leur passé), la technologie utilisée par la production elle-même se doit de simuler des prises de vue correspondant aux possibilités technologiques actuelles, renforçant encore l'effet-réalité. On voit cependant ici à quel point celui-ci peut relever de l'effet spécial et être arbitraire, ressortissant plus du domaine de l'illusion que de l'authentique référentialité.

\section{Les réalités de la science-fiction}

L'effet-réalité consiste enfin à faire croire, non seulement à la possibilité de voir les événements ou phénomènes montrés à l'écran survenir un jour dans la réalité, comme c'est le cas dans l'extrapolation, mais aussi de faire croire à une réalité, celle du monde mis en scène. L'effet-réalité consiste alors à créer un monde, une réalité, à la fois autre et spéculaire.

L'effet passe ici par la description narrative des institutions du monde imaginaire décrit, son histoire, sa géographie, sa structure sociale, ses religions et ses coutumes, ses langues, etc., qu'opèrent savamment Babylon 5 et Galactica au cours de leurs saisons. Ce « réalisme » particulier, consistant en la re-construction d'une réalité, tient principalement des séries elles-mêmes mais aussi de leurs téléfilms et de leurs divers "spin-offs » (Caprica et bientôt peut-être Blood \& Chrome, tous deux des «prequels » de Galactica), le film actuellement en pré-production, les romans et graphic novels, la websérie, les jeux vidéos, etc. La série reproduit alors le phénomène déjà observé au cinéma depuis les années quatre-vingt (Star Wars) et à la télévision depuis les années quatre vingt-dix (B5 mais aussi Star Trek), ainsi que dans les œuvres fleuves de science-fiction et de fantasy contemporaines, où chaque nouveau volume contribue à élargir un peu plus un univers fictionnel en constante expansion, tandis que le paratexte de ces œuvres vient typiquement renforcer son propre effet de réel par l'intermédiaire de cartes, d'un alphabet ou de chroniques historiques.

De même, cette construction progressive d'un univers et d'un réel alternatif est rendue possible par l'usage fait, pour la première fois en SF par Babylon 5 puis par Galactica, de la même narration 
feuilletonnante ${ }^{23}$, caractéristique de la renaissance des séries TV, plus particulièrement depuis le début des années deux mille. Plutôt que vaincre un nouvel obstacle hebdomadaire comme c'était autrefois le cas, la nouvelle science-fiction télévisuelle s'axe sur des intrigues qui se nouent progressivement au fil de nombreux épisodes voire de ses différentes saisons (et parfois même d'un média à l'autre, comme dans le cas de la websérie, qui s'articule entre les saisons deux et trois de la série-mère), se résolvent ou se complexifient, élaborant lentement ce que l'on en est venu à appeler aujourd'hui, principalement depuis Twin Peaks et The $X$-files, une "mythologie » construite progressivement par une série et ses déclinaisons transmédiatiques. Galactica emprunte même à $B 5$ son idée (elle est la première à la mettre en œuvre) de produire, pendant ou même après la fin de la production de la série, des téléfilms qui se situent chronologiquement entre deux épisodes ou deux saisons de celle-ci (ce que l'on tend à appeler aujourd'hui, sur le modèle des anglicismes « sequel » et « prequel », un « interquel 24 »), afin à nouveau de développer l'univers et la mythologie - la réalité - de la série. L'évolution est particulièrement frappante dans le cas de Star Trek, qui passe, durant les années quatre vingt-dix dans le spin-off Deep Space Nine et sous l'influence directe de Babylon 5, du schéma de la "planète de la semaine " à celui d'un véritable feuilleton qui développe des intrigues et un univers de plus en plus complexe et cohérent. Ronald Moore est précisément un des producteurs et scénaristes de Deep Space Nine (cf. note 11).

$\mathrm{Si}$ d'autres genres connaissent le même développement, la science-fiction, qui a vocation (avec la fantasy) à créer des mondes imaginaires, semble à nouveau se prêter naturellement et structurellement à ce développement. C'est ce qu'Anne Besson, à la suite de Richard St. Gelais, nomme le «xéno-encyclopédisme » caractéristique de la fantasy et de la science-fiction, désignant ainsi le processus de lecture (ou de visionnage) spécifique à la découverte progressive du monde ou de l'univers typiquement proposé par le texte, « la promesse, d'essence structurelle, d'un univers concurrent du réel $[\ldots]^{25}$ ». La forme sérielle se prête idéalement à une telle " obsession démiurgique ${ }^{26}$ », menant à la fois à créer une réalité autre dans laquelle s'abandonner avec délices chaque semaine, dont la découverte progressive participe du plaisir de visionnage et de lecture, et à créer un monde spéculaire qui renvoie potentiellement à notre réalité, à son actualité et/ou son organisation et ses institutions

23 C'est-à-dire, pour reprendre la définition donnée par Alain Carrazé, une «sériefeuilleton » ou une "série feuilletonnante », "[...] qui, en plus de raconter des histoires complètes, se lan[cent] aussi dans des trames 'à suivre' au fil des épisodes [...]» (Alain Carrazé, Les séries télé, Paris, Hachette, 2007, édition kindle).

24 <http://tvtropes.org/pmwiki/pmwiki.php/Main/Interquel>, consulté le 02/05/2011.

${ }^{25}$ Anne Besson, La fantasy, Paris, Klincksieck, 2007, p. 23, 94

${ }^{26}$ André-François Ruaud, Cartographie du merveilleux. Paris, Editions Denoël, 2001, p. 35 
sociales. Le "réalisme » en science-fiction tend alors à recouper les notions de vraisemblance et de cohérence discursive d'un univers entièrement imaginaire, le résultat idéal étant d'emporter l'adhésion du téléspectateur quant au monde qu'il a sous les yeux et quant à la « vérité » profonde des personnages (l'effet-réalité est donc bien pensé comme un effet-vérité), ainsi que de l'inviter idéalement à la réflexion sur ses propres convictions politiques, sociales et religieuses et la façon dont le monde réel fonctionne.

A côté de la réalité existent donc des réalités imaginaires, et tout le talent des intrigues et de la mise en scène (quelle que soit l'esthétique précise qu'elle adopte) consiste à nous faire croire en la réalité spéculative qui nous est décrite dans l'œuvre en question. Le même phénomène se vérifie dans toute série TV, indépendamment de son genre d'appartenance, et c'est pourquoi on peut considérer ici aussi la science-fiction comme un genre emblématique des séries télévisées dans leur ensemble, mettant en évidence ce qui se produit de façon plus souterraine dans les autres genres : chaque série consiste en la plongée et en «l'immersion » (terme qui revient souvent sous la plume des critiques et des journalistes) du téléspectateur dans son univers particulier, quel que soit le genre précis de la série en question. L'effetréalité et la notion de réalisme consistent donc ici à créer et à construire de façon convaincante une réalité particulière, en l'occurrence un monde, un univers parallèle, ce que fait littéralement la $\mathrm{SF}$, au caractère ludique duquel peut s'ajouter une dimension spéculaire de « retour » vers le monde réel, voire de critique de certains aspects de celui-ci.

La narration feuilletonnante permet enfin à la série de se concentrer sur ses personnages, leurs relations et alliances évanescentes et surtout leurs faiblesses et l'évolution qui est la leur au fil des saisons. Les personnages de $B 5$ étaient déjà bien plus sombres que les acteurs du space opera traditionnel, et préfiguraient les protagonistes de Galactica. Pour la première fois, Babylon 5 mettait en effet en scène des personnages dotés de faiblesses, joueurs, menteurs, manipulateurs, voire parfois alcooliques et violents, presque parfois des antihéros dont le combat moral interne représentait une part importante des intrigues de la série, ce qui est également le cas de Galactica. Garibaldi, le chef de la sécurité de la station Babylon, est par exemple un alcoolique notoire qui doit affronter le démon de la bouteille, tout comme le colonel Tigh de Galactica. Le Dr. Franklin de $B_{5}$, qui s'est engagé pour imiter son général de père, entretient une relation difficile avec celui-ci, tous deux ayant de forts caractères et se ressemblant un peu trop pour leur propre bien, schéma que l'on retrouve dans Galactica avec les Adama père et fils. Le commandant Sheridan de Babylon 5, le personnage central de la première saison, est hanté par le souvenir de sa femme décédée, ce qui s'avèrera être également partiellement le cas de l'amiral Adama. 
C'est aussi ce qu'a en tête Ron Moore lorsqu'il qualifie sa série de naturaliste : il cherche à se focaliser sur des personnages dont la réalité (c'est-à-dire aussi leurs défauts et leurs faiblesses, leurs mensonges et leurs tromperies) serait fidèlement rendue. Ce type de personnage s'est multiplié à partir des années quatre vingt-dix dans divers genres (le Sipowicz de NYPB Blue s'est imposé comme l'exemple paradigmatique du phénomène), et cet aspect sombre et torturé se prête bien à l'atmosphère dystopique du nouveau space opera. Il rompt ainsi avec l'atmosphère bonne enfant qui régnait traditionnellement dans les séries du genre, puisque les conflits parfois très violents entre les principaux personnages constituent une part importante des intrigues de $B 5$ et Galactica. Comme l'écrit Moore dans son essai :

Notre série est avant tout une série dramatique [ " a drama »].
Elle parle avant tout d'individus. De vrais gens auxquels le
public peut s'identifier et dans lesquels il peut s'investir. Ce
n'est pas une série centrée sur la technologie ou d'étranges
cultures extraterrestres. C'est une série sur nous, l'allégorie de
notre propre société et nous-mêmes, ce qui devrait être
immédiatement clair pour chaque téléspectateur.

\section{Conclusion}

Babylon 5 et Galactica sont exemplaires du fonctionnement structurel de la science-fiction, mais aussi d'une grande partie des séries télévisées contemporaines: divertir le téléspectateur en l'emportant dans une réalité imaginaire totalisante dont on nous décrit et explicite les aspects aussi bien politiques, historiques, religieux, économiques ou sociaux; mais aussi très souvent le ramener à la réalité initiale, notre réalité (ou la réalité spécifique de chaque téléspectateur), l'aperçu globalisant offert au téléspectateur omniscient lui permettant de donner du sens à une réalité et un présent souvent perçus de manière fragmentée et partielle. L'effet-réalité a donc toute sa place dans une science-fiction qui n'est jamais aussi signifiante que lorsque $s a$ réalité est fermement établie et installée, quand on peut non seulement «suspendre son incrédulité » mais aussi adhérer au " réalisme » de ce monde secondaire. Le réalisme devient double, et répond à une dynamique entre l'effet-réalité et « l'effet d'altérité », qui fait tout l'attrait de la science-fiction. C'est pourquoi Isaac Asimov pouvait déjà écrire que "la science-fiction est une évasion dans la réalité » (" an escape into reality ${ }^{27}$ »). La science-fiction a tout à voir avec le réel - avec tous les réels.

${ }_{27}$ Cité in James Gunn, Inside Science Fiction. Essays on Fantastic Literature. San Bernardino, Borgo Press, 1992, p. 152. 